\title{
Identification of Mass Parameter of Non-Cooperative Space Target based on Vibration Characteristic of Flexible-joint Manipulator
}

\author{
Yanhua Han a , Xinyu $\mathrm{Li}^{\mathrm{b}}$, \\ Nanjing University of Aeronautics and Astronautics, Nanjing 210016, China. \\ ahanyanhua@nuaa.edu.com, ba1392942175@163.com
}

\begin{abstract}
For the identification of mass parameter of non-cooperative space target in on-orbit servicing missions, a method of mass identification based on vibration characteristic of the flexible-joint manipulator is proposed. Firstly, the flexible-joint manipulator captures non-cooperative target. Then, the dynamic model of the combined-body formed by operational spacecraft, flexible-joint manipulator and non-cooperative space target is established. Finally, the mass of non-cooperative space target is identified by analyzing the vibration frequency of the manipulator. Numerical simulation results show the effectiveness of the method.
\end{abstract}

Keywords: Flexible-joint manipulator; non-cooperative space target; dynamic modeling; mass identification.

\section{Introduction}

As the target of customer system, the spacecraft can be divided into cooperative and non-cooperative [1] in space on-orbit servicing missions. In general, the space cooperative target is considered as the spacecraft that can provide mass characteristic parameters and other information to another spacecraft. The on-orbit servicing technology of space cooperative target has been more and more mature and successfully applied to the rendezvous and docking of space station and other fields, such as the SMART-OLEV project [2] of the European Space Agency, the ETS-VII project [3] of Japan. Space non-cooperative target refers to the space objects with unknown mass characteristic parameters, including fault satellites, space debris and enemy spacecrafts [4], etc. There is no information exchange between the non-cooperative target and the servicing spacecraft, which may cause harmful effects on the high precision attitude control of the combined-body. Therefore, it is necessary to identify mass parameter of the non-cooperative target accurately.

At present, domestic and foreign scholars have conducted a lot of research on the mass identification of non-cooperative target and the combined-body. Some identification methods have been put forward. Murotsu [5] researched on the parameter identification problem of the space robot capturing unknown target. An identification method based on the conservation principle of linear momentum was proposed. However, this method can only identify the inertial parameters of the combined-body and cannot identify the mass of the unknown target. Yoshida[6] researched on the problem of flywheel angular momentum error, presented a method based on the principle of conservation of angular momentum to calculate the mass parameter of space robot and links. Wang Ming [7] proposed a method for on-orbit identification of inertial parameters of compound spacecraft using space manipulator. This method can be decomposed into two steps. The first step is to identify mass and mass center of the compound spacecraft. The second step is to identify inertia tensor of the compound spacecraft based on the principle of conservation of momentum. Zhang Fan [8] used the space tethered robot to catch the unknown target and proposed a method using the vibration characteristics of the stage after capturing to identify the parameters of unknown target. Firstly, the non-dimensional equations of motion of the system are derived as two rigid bodies. Then, the attitude motions of target satellite and tether system during the post-capture phase are analyzed. Finally, the

Inertia parameters of non-cooperative target satellite are estimated by using a robust variable forgetting factor recursive least-squares algorithm under the condition that all the information of target is not communicated to the base spacecraft. Zhang Haibo [9] considered the space robot and non-cooperative target as a whole system. The total mass properties were identified after 
non-cooperative target being captured by the space robot by using the recursive least squares method. Li Dongbai [10] researched on the identification of mass and centroid position of the satellite in on-orbiting servicing after capturing the target and put forward a method for the identification of mass and centroid using least square method. Jin Lei [11] used the free-floating robot to capture the unknown target. This method can be divided into two steps. The first step is to establish dynamic model of the manipulator and the unknown target in inertial coordinate system. The second step is to measure linear velocity, angular velocity and joint angle of the manipulator at different times. The inertial parameters can be accurately identified by using this method in both cases of zero and non-zero momenta and the problem of singular solution can also be avoided.

Most of the researches above on non-cooperative target mass identification are based on the conservation of momentum or the least square method. The joint flexibility of the manipulator is ignored. This paper aims at the coupled rigid-flexible combined-body system and presents a method of mass identification based on vibration characteristic of flexible-joint manipulator. Numerical simulation results show the effectiveness of the method.

\section{Dynamic Model of Flexible-joint Manipulator and Non-cooperative Target}

\subsection{Problem Description.}

In general, as reference to the world's SMART-OLEV and DEOS projects, the combined-body system includes the operational spacecraft, the space manipulator and the non-cooperative target, as shown in Fig.1.

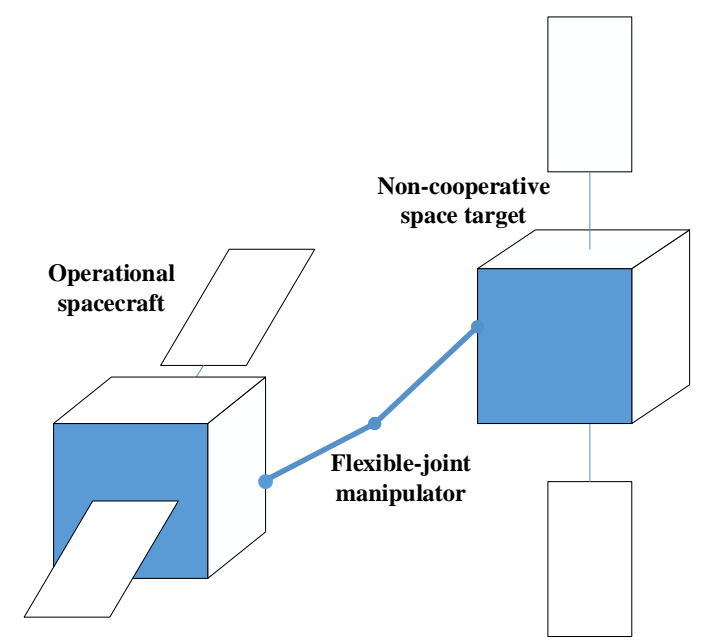

Fig. 1. Illustration of the combined-body

\subsection{Dynamic Model of the Combined-body.}

In order to identify the mass of non-cooperative target, the space coordinate system $O x y z$ is assumed as the inertial coordinate system. The body coordinate system $O_{0} x_{0} y_{0} z_{0}$ is established at the centroid of the operational spacecraft. Plane $O_{0} x_{0} y_{0}$ and plane $O x y$ are parallel. Point A is the hinge point of the manipulator and the operational spacecraft. $O_{0} x_{0}$ axis passes through the $O_{0}$ point and points to point A. $O_{0} y_{0}$ axis is obtained by rotating $O_{0} x_{0}$ axis with 90 degrees in counter clockwise. $O_{0} z_{0}$ axis is determined by the right hand rule. This paper only studies the motion of the spacecraft in plane $O x y$, so the motion in the $z$ axis is ignored. Assuming that the non-cooperative target is a particle, Fig. 2 is a simplified model of the combined-body system formed by the operational spacecraft and non-cooperative target. 


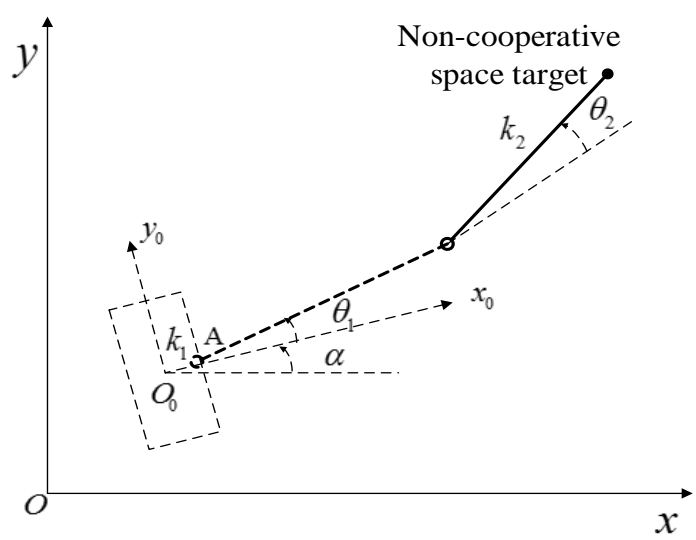

Fig.2. Simplified model of the combined-body system

Assuming that the mass of the operational spacecraft is $m$, the moment of inertia regarding to centroid of the spacecraft is $J$. In the inertial coordinate system, the centroid position of operational spacecraft is $(x, y)$. The angle between $O x$ axis and $O_{0} x_{0}$ axis is $\alpha . \alpha$ is the pitch angle of the operational spacecraft. The two links of the manipulator are uniform beans. Mass of each link is $m_{1}$ and $m_{2}$. Length of each link is $l_{1}$ and $l_{2}$. The moment of inertia regarding to each centroid of link is $J_{1}$ and $J_{2}$. Joints of two links are flexible. The stiffness coefficient of the two hinge points is $k_{1}$ and $k_{2}$, respectively. The end position of link 2 is fixed with a non-cooperative target with unknown mass expressed as $m_{3}$. Rotation angle of the elastic deflection of link 1 and link 2 due to the flexibility of the joint is $\theta_{1}$ and $\theta_{2}$.

In the inertial coordinate system, the coordinate of centroid position of the non-cooperative target is shown in Eq. (1):

$$
\left\{\begin{array}{l}
x_{3}=x+l_{1} \cos \left(\alpha+\theta_{1}\right)+l_{2} \cos \left(\alpha+\theta_{1}+\theta_{2}\right) \\
y_{3}=y+l_{1} \sin \left(\alpha+\theta_{1}\right)+l_{2} \sin \left(\alpha+\theta_{1}+\theta_{2}\right)
\end{array}\right.
$$

The position of centroid of the link 1 is shown in Eq. (2) (neglecting the distance between the space coordinate system $O_{0}$ and the hinge point):

$$
\left\{\begin{array}{l}
x_{1}=x+\frac{1}{2} l_{1} \cos \left(\alpha+\theta_{1}\right) \\
y_{1}=y+\frac{1}{2} l_{1} \sin \left(\alpha+\theta_{1}\right)
\end{array}\right.
$$

The position of centroid of the link 2 is shown in Eq. (3):

$$
\left\{\begin{array}{l}
x_{2}=x+l_{1} \cos \left(\alpha+\theta_{1}\right)+\frac{1}{2} l_{2} \cos \left(\alpha+\theta_{1}+\theta_{2}\right) \\
y_{2}=y+l_{1} \sin \left(\alpha+\theta_{1}\right)+\frac{1}{2} l_{2} \sin \left(\alpha+\theta_{1}+\theta_{2}\right)
\end{array}\right.
$$

Translational kinetic energy of the operational spacecraft is shown in Eq. (4):

$$
T_{\mathrm{P}}=\frac{1}{2} m\left(\dot{x}^{2}+\dot{y}^{2}\right)
$$

Translational kinetic energy of the link 1 and 2 are shown in Eq. (5) and (6):

$$
\begin{aligned}
& T_{\text {IP }}=\frac{1}{2} m_{1}\left(\dot{x}_{1}^{2}+\dot{y}_{1}^{2}\right) \\
& T_{\text {IIP }}=\frac{1}{2} m_{2}\left(\dot{x}_{2}^{2}+\dot{y}_{2}^{2}\right)
\end{aligned}
$$

Rotational kinetic energy of the operational spacecraft is shown in Eq. (7): 


$$
T_{\mathrm{Z}}=\frac{1}{2} J \cdot \dot{\alpha}^{2}
$$

Rotational kinetic energy of the link 1 and 2 are shown in Eq. (8) and Eq. (9):

$$
\begin{aligned}
& T_{\mathrm{IZ}}=\frac{1}{2} J_{1}\left(\dot{\alpha}+\dot{\theta}_{1}\right)^{2} \\
& T_{\mathrm{IIZ}}=\frac{1}{2} J_{2}\left(\dot{\alpha}+\dot{\theta}_{1}+\dot{\theta}_{2}\right)^{2}
\end{aligned}
$$

The kinetic energy of the non-cooperative target is shown in Eq. (10):

$T_{3}=\frac{1}{2} m_{3}\left(\dot{x}_{3}^{2}+\dot{y}_{3}^{2}\right)$

The total kinetic energy of the system is shown in Eq. (11):

$T=T_{\mathrm{P}}+T_{\mathrm{IP}}+T_{\mathrm{IIP}}+T_{\mathrm{Z}}+T_{\mathrm{IZ}}+T_{\mathrm{IIZ}}+T_{3}$

The elastic potential energy at the hinge point are shown in Eq. (12) and Eq. (13):

$V_{1}=\frac{1}{2} k_{1} \theta_{1}^{2}$

$V_{2}=\frac{1}{2} k_{2} \theta_{2}^{2}$

The total potential energy of the system is shown in Eq. (14):

$V=V_{1}+V_{2}$

Lagrange function is shown in Eq. (15):

$L=T-V$

System generalized coordinates:

$q_{1} \square x, q_{2} \square y, q_{3} \square \alpha, q_{4} \square \theta_{1}, q_{5} \square \theta_{2}$

The generalized force of the system is zero, that is:

$Q_{1}=Q_{2}=Q_{3}=Q_{4}=Q_{5}=0$

Substituting the Eq. (4) (14) into the Eq. (15) and substituting Eq. (15) (16) into Lagrange equation:

$$
\left(\frac{\mathrm{d}}{\mathrm{d} t} \frac{\partial}{\partial \dot{q}_{j}}-\frac{\partial}{\partial q_{j}}\right) L=Q_{j}, j=1,2 \cdots 5
$$

Dynamic equation of the system can be obtained:

$M \ddot{q}+C \dot{q}+K q=0(18)$

Mass matrix, damping matrix and stiffness matrix are listed below:

$M \square\left(\begin{array}{lllll}m_{11} & m_{12} & m_{13} & m_{14} & m_{15} \\ m_{21} & m_{22} & m_{23} & m_{24} & m_{25} \\ m_{31} & m_{32} & m_{33} & m_{34} & m_{35} \\ m_{41} & m_{42} & m_{43} & m_{44} & m_{45} \\ m_{51} & m_{52} & m_{53} & m_{54} & m_{55}\end{array}\right), C \square\left(\begin{array}{lllll}c_{11} & c_{12} & c_{13} & c_{14} & c_{15} \\ c_{21} & c_{22} & c_{23} & c_{24} & c_{25} \\ c_{31} & c_{32} & c_{33} & c_{34} & c_{35} \\ c_{41} & c_{42} & c_{43} & c_{44} & c_{45} \\ c_{51} & c_{52} & c_{53} & c_{54} & c_{55}\end{array}\right), K \square\left(\begin{array}{lllll}k_{11} & k_{12} & k_{13} & k_{14} & k_{15} \\ k_{21} & k_{22} & k_{23} & k_{24} & k_{25} \\ k_{31} & k_{32} & k_{33} & k_{34} & k_{35} \\ k_{41} & k_{42} & k_{43} & k_{44} & k_{45} \\ k_{51} & k_{52} & k_{53} & k_{54} & k_{55}\end{array}\right)$

\section{The Mass Identification Method of Non-cooperative Target}

In this paper, the characteristic frequency of the vibration of the flexible-joint manipulator is used to identify the mass of the non-cooperative target.

When the manipulator is in the balance position, the joint angle is $\theta_{1}=\theta_{2}=0$. The system dynamic equation is linearized with small perturbation in the balance position of the manipulator.

$$
\bar{M} \ddot{q}+\bar{C} \dot{q}+\bar{K} q=0
$$

Where, the mass matrix, damping matrix and stiffness matrix are: 


$$
\bar{M} \square\left(\begin{array}{lllll}
\bar{m}_{11} & \bar{m}_{12} & \bar{m}_{13} & \bar{m}_{14} & \bar{m}_{15} \\
\bar{m}_{21} & \bar{m}_{22} & \bar{m}_{23} & \bar{m}_{24} & \bar{m}_{25} \\
\bar{m}_{31} & \bar{m}_{32} & \bar{m}_{33} & \bar{m}_{34} & \bar{m}_{35} \\
\bar{m}_{41} & \bar{m}_{42} & \bar{m}_{43} & \bar{m}_{44} & \bar{m}_{45} \\
\bar{m}_{51} & \bar{m}_{52} & \bar{m}_{53} & \bar{m}_{54} & \bar{m}_{55}
\end{array}\right), \bar{C} \square\left(\begin{array}{lllll}
\bar{c}_{11} & \bar{c}_{12} & \bar{c}_{13} & \bar{c}_{14} & \bar{c}_{15} \\
\bar{c}_{21} & \bar{c}_{22} & \bar{c}_{23} & \bar{c}_{24} & \bar{c}_{25} \\
\bar{c}_{31} & \bar{c}_{32} & \bar{c}_{33} & \bar{c}_{34} & \bar{c}_{35} \\
\bar{c}_{41} & \bar{c}_{42} & \bar{c}_{43} & \bar{c}_{44} & \bar{c}_{45} \\
\bar{c}_{51} & \bar{c}_{52} & \bar{c}_{53} & \bar{c}_{54} & \bar{c}_{55}
\end{array}\right), \bar{K} \square\left(\begin{array}{lllll}
\bar{k}_{11} & \bar{k}_{12} & \bar{k}_{13} & \bar{k}_{14} & \bar{k}_{15} \\
\bar{k}_{21} & \bar{k}_{22} & \bar{k}_{23} & \bar{k}_{24} & \bar{k}_{25} \\
\bar{k}_{31} & \bar{k}_{32} & \bar{k}_{33} & \bar{k}_{34} & \bar{k}_{35} \\
\bar{k}_{41} & \bar{k}_{42} & \bar{k}_{43} & \bar{k}_{44} & \bar{k}_{45} \\
\bar{k}_{51} & \bar{k}_{52} & \bar{k}_{53} & \bar{k}_{54} & \bar{k}_{55}
\end{array}\right)
$$

According to the linear system theory, the eigenvalue of matrix $\bar{M}^{-1} \cdot \bar{K}$ is the square of the characteristic frequency of the system. That is:

$$
\omega^{2}=\operatorname{eig}\left(\bar{M}^{-1} \cdot \bar{K}\right)
$$

Table 1 lists the parameters of the system.

Table 1 Parameters of the system

\begin{tabular}{ccc}
\hline Parameter & Unit & Value \\
\hline$m$ & $\mathrm{~kg}$ & 20 \\
$m_{1}$ & $\mathrm{~kg}$ & 1 \\
$m_{2}$ & $\mathrm{~kg}$ & 1 \\
$m_{3}$ & $\mathrm{~kg}$ & 5 \\
$l_{1}$ & $\mathrm{~m}$ & 1 \\
$l_{2}$ & $\mathrm{~m}$ & 1 \\
$J$ & $\mathrm{~kg} \cdot \mathrm{m}^{2}$ & 10 \\
$J_{1}$ & $\mathrm{~kg} \cdot \mathrm{m}^{2}$ & 10 \\
$J_{2}$ & $\mathrm{~kg} \cdot \mathrm{m}^{2}$ & 10 \\
$k_{1}$ & $\mathrm{~N} \cdot \mathrm{m}$ & 900 \\
$k_{2}$ & $\mathrm{~N} \cdot \mathrm{m}$ & 900 \\
\hline
\end{tabular}

According to the parameters in table 1, the eigenvalues of the system are:

$$
\left\{\begin{array}{l}
s_{1}=\frac{1341045 m_{3}+6961500-45 \sqrt{29\left(9766469 m_{3}^{2}+86566840 m_{3}+196034000\right)}}{8401 m_{3}+40100} \\
s_{2}=\frac{1341045 m_{3}+6961500+45 \sqrt{29\left(9766469 m_{3}^{2}+86566840 m_{3}+196034000\right)}}{8401 m_{3}+40100}
\end{array}\right.
$$

The two eigenvalues are all positive, so it is feasible to calculate the characteristic frequency of the vibration of the system.

In this paper, MATLAB is used to analyze the vibration of the manipulator and calculate the characteristic frequency of vibration. Then the frequency is substituted into the Eq. (21) to calculate the mass of non-cooperative target. The calculated mass is compared with the actual mass to verify the effectiveness of the proposed method.

The numerical simulation of the vibration of the manipulator is performed. The simulation entry parameters are shown in table 1.

The MATLAB simulation results are:

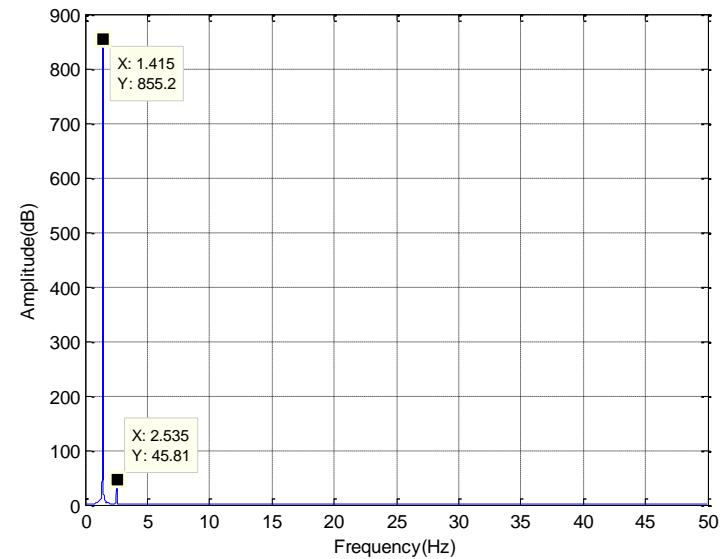

Fig. 3. Illustration of characteristic frequency of the manipulator's vibration when $m_{3}=5 \mathrm{~kg}$ 
By fast Fourier transformation, the first two order characteristic frequencies of the vibration of the manipulator are $f_{1}=1.415 \mathrm{~Hz}$ and $f_{2}=2.535 \mathrm{~Hz}$. The corresponding angular frequency is $\omega_{1,2}=2 \pi f_{1,2}$. Then $s_{1,2}=\omega_{1,2}{ }^{2}$ is substituted into Eq. (21) and the mass identification parameter of non-cooperative target $m_{3}^{*}$ will be resolved.

The solution is $m_{3}^{*}=5.211 \mathrm{~kg}$ based on the first-order frequency. And the relative error is $4.22 \%$. The solution is $m_{3}^{*}=-4.918 \mathrm{~kg}$ based on the second-order frequency and can be dropped.

To change the mass of non-cooperative target without changing the other parameters of the system and using the above method to identify the mass. The mass of non-cooperative targets were $8 \mathrm{~kg}, 10 \mathrm{~kg}$, $15 \mathrm{~kg}$ and $20 \mathrm{~kg}$, respectively. Fig. $4-7$ are the simulation results of the vibration characteristic frequency of flexible-joint manipulator with different mass.

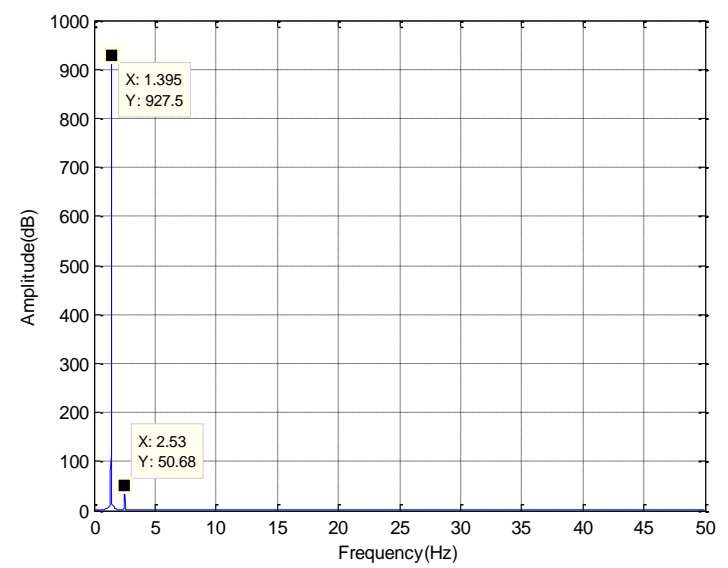

Fig. 4 Illustration of Characteristic Frequency of the Manipulator's Vibration When $m_{3}=8 \mathrm{~kg}$

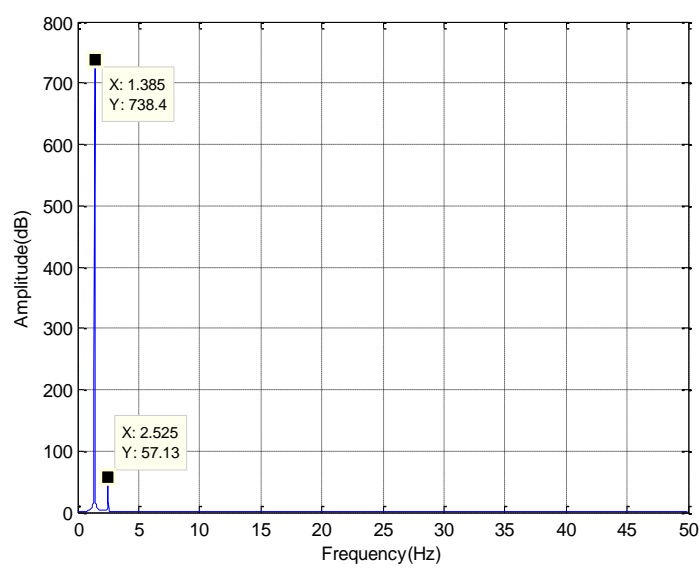

Fig. 5 Illustration of Characteristic Frequency of the Manipulator's Vibration When $m_{3}=10 \mathrm{~kg}$

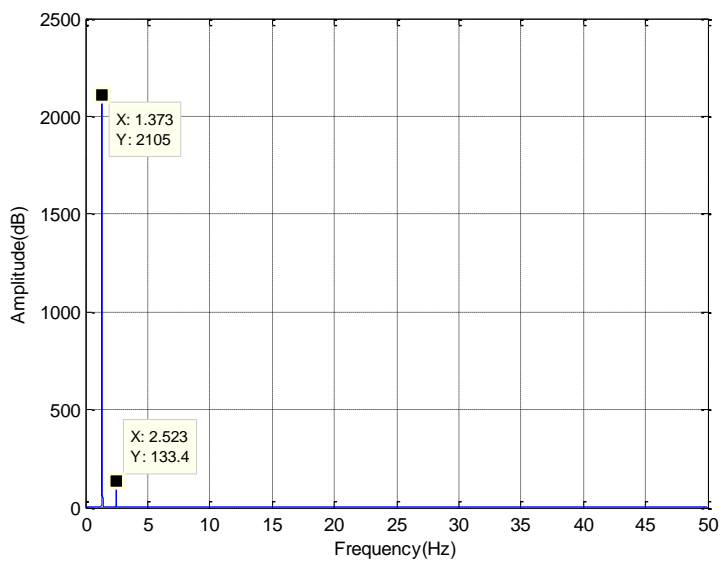

Fig. 6 Illustration of Characteristic Frequency of the Manipulator's Vibration When $m_{3}=15 \mathrm{~kg}$ 


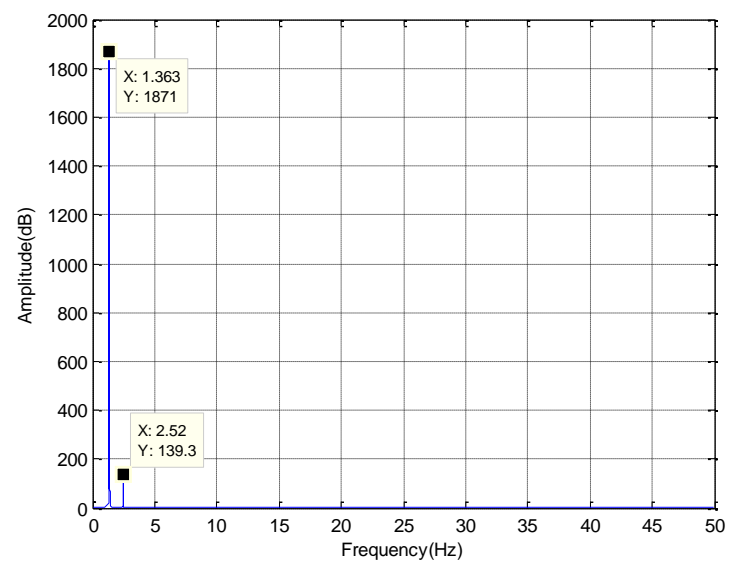

Fig. 7 Illustration of Characteristic Frequency of the Manipulator's Vibration When $m_{3}=20 \mathrm{~kg}$

The feasibility and effectiveness of the proposed method are illustrated by identifying non-cooperative target with different mass, as shown in table 2.

Table 2 Results of the identification

\begin{tabular}{cccc}
\hline$m_{3} / \mathrm{kg}$ & $f_{1} / \mathrm{Hz}$ & $m_{3}^{*} / \mathrm{kg}$ & Relative error \\
\hline 5 & 1.415 & 5.211 & $4.22 \%$ \\
8 & 1.395 & 8.293 & $3.66 \%$ \\
10 & 1.385 & 10.627 & $6.27 \%$ \\
15 & 1.373 & 14.757 & $1.62 \%$ \\
20 & 1.363 & 20.282 & $1.41 \%$ \\
\hline
\end{tabular}

From table 2, we can see that the proposed method shows a better performance for the mass identification of non-cooperative target and the error is kept small, which is acceptable in engineering.

\section{Summary}

In this paper, the coupled rigid-flexible combined-body system of operational spacecraft and space non-cooperative target with flexible-joint manipulator is studied. Aiming at mass identification of non-cooperative target, a method based on the flexible-joint manipulator vibration frequency to identify the mass of space non-cooperative target is proposed.

This method only requires small angle vibration of manipulator and records the vibration by sensor. Then the characteristic frequency of vibration is obtained by means of the fast Fourier transformation. Finally, mass of non-cooperative target can be calculated.

Simulation results show that the proposed method is feasible and can be used in future non-cooperative object mass identification.

\section{References}

[1] Liang B, Du X D, Li C, et al. Advances in Space Robot On-orbit Servicing for Non-cooperative Spacecraft. Robot. Vol. 34(2012) No. 2, p. 242-256.

[2] Kaiser C, Sjoberg F, Delcura J M, et al. SMART-OLEV-An orbital life extension vehicle for servicing commercial spacecrafts in GEO. Acta Astronautics. Vol. 63(2008) p. 400-410.

[3] Oda M, Fukushima N I Y. Space robot technology experiments on NASDA's ETS-VII satellite. Advanced Robotics the International Journal of the Robotics Society of Japan, 1999, p. 335-336.

[4] Kassebom M. ROGER - An Advanced Solution for a Geostationary Service Satellite. Robot. Vol. 35(2003) No. 2, p. 216-225.

[5] Murotsu Y, Senda K, Ozaki M. Parameter identification of unknown object handled by free-flying space robot. Journal of Guidance Control \& Dynamics. Vol. 1(1992) No. 3, p. 488-494. 
[6] Yoshida K. Inertia parameter identification for a free-flying space robot. AIAA Journal. Vol. 68(2002) No. 62, p. 2002-4568.

[7] Wang M, Huang P F, Chang $\mathrm{H}$ T. Identification of mass characteristic parameters for non-cooperative target spacecraft. Flight Dynamics. Vol. 32(2014) No. 6, p. 201-212.

[8] Zhang F, Huang P f. Inertia parameter estimation for an non-cooperative. Journal of Astronautics. Vol. 36(2015) No. 6, p. 630-639.

[9] Zhang H B, Wang D Y, Wei C L. On-orbit identification of mass properties of non-cooperative space target. Aerospace Control. Vol. 33(2015) No. 3, p. 23-28.

[10] Li D B, Liu C, Wang F. Method of Mass and Centroid Identification for User Satellites. Proceedings of the 2013 Conference on space optics and mechanical and electrical technology, China Space Science Society.

[11] Jin L, Xu S J. Inertial parameter identification of unknown object captured. Journal of Astronautics. Vol. 33(2012), p. 1570-1576. 